\title{
Role of Requirement Management as a CMMI Process Area in Requirement Engineering
}

\author{
Samina Jan \\ Department of Software Engineering \\ Faculty of Telecommunication and Information \\ Engineering \\ University of Engineering \& Technology Taxila, \\ Pakistan
}

\author{
Muhammad Nadeem Majeed \\ Department of Software Engineering \\ Faculty of Telecommunication and Information \\ Engineering \\ University of Engineering \& Technology Taxila, \\ Pakistan
}

\begin{abstract}
The research shows role of requirement management as a CMMI process area in requirement engineering (a phase of software engineering). Requirement engineering (RE) activity consists of several activities like requirement elicitation by feasibility studies, requirement analysis, requirement validation and requirement management. While performing RE, recognized formal standards should follow like CMMI, ISO etc. Proposed work helps in RE process by acquiring CMMI requirement management process area at level 2 .

There is confusion in the minds of software personnel between the requirements management process area in CMMI and manage requirements activity in the requirement engineering. So they don't consider requirement management process area as development improvement approach, they think that we don't need anything which requires explicit methods to apply on existing process. Mistake leads to inefficient requirement engineering. The study gives an idea about importance of requirement management while gathering, analyzing and implementing the requirements which are given by the stakeholders. Sometimes stakeholders don't know about their actual requirements and due to this, misunderstanding takes place which brings dissatisfaction of stakeholders and ultimately results poor reputation of organizations.
\end{abstract}

\section{General Terms}

et. al.

\section{Keywords}

CMMI, process area, requirement management, requirement engineering

\section{INTRODUCTION}

It is necessary to understand the requirements. Requirements are described in two forms. Enduring or stable requirements are the core functionalities of the system or related to the domain area. And the other one are volatile requirements which alter during development process or after deployment of the system. It is impossible to keep the requirements stable till the end of the development process, so volatile requirements mostly exist. Volatile requirements are classified in different categories. Mutable requirements category consist of those requirements which are changing according to the environment of the system, environment in which the system is deployed and working. Emergent requirements category depends on the understanding of the system developers, how they understand the system at initial level, so when the system requirements were not correctly specified at the design stage or requirements emerge after the system implementation. Consequential requirements category is result of requirement understanding process, we assume at initial stage that how the system will work or operate, but when system comes into deployment or in operational stage, the system perform opposite to our assumptions, so the requirements need to change. Compatibility requirements category relays on the other requirements, systems or organizations policies and processes. Compatibility requirements are sort of constraints which should kept in mind during the development of the system like one should consider that the requirements are compatible with the required process or equipment.

\section{LITERATURE REVIEW}

$X$. Wang et al[1] worked on software requirements management at organizational level. They suggested that requirements can be manage at organizational level by dividing them into different categories. Three Categories are the customer requirement management, product requirement management and management of developing requirements. Also they mentioned the objects of requirement management at organizational stage. There are three main components which will come under management requirement.

1. Business requirement suggestions consist of four states. First is "Null" (suggestions about the requirement have not yet been evaluated). Second state is "Rejected" (suggestions about the requirement have been evaluated but been rejected).Third state is "Approved" (suggestions about the requirement have been evaluated and have been approved).Fourth state is "Accept and hold in abeyance" (suggestions about the requirement have been evaluated but temporary disuse or suspension).

2. Product development requirements consist of four states. First is "Null" (a default state). Second state is "Already put in storage" (suggestions about the requirement have been evaluated and specifications of requirements also written).Third state is "Being Developed" (suggestions about the requirement have been evaluated and have been approved for development).Fourth state is "Released" (product or service has been deployed). 


\section{Product specifications}

Specification objects pass on for the development of single function or feature.

Justin J.Y. Lin et al [2] verified that the requirement management (REQM) in CMMI (Capability Maturity Model Integration) is an efficient way that tells how to tackle the requirements and internationally it is proved that requirement management process area is the best guide for implementing requirement in real life while doing different projects. They said that it is not only cover the analysis, refined, measure and manage the requirements but also helpful in maintaining different types of documents that are unpredictable and inevitable. Yufei Xie et al [3] worked on the complex systems. They provide solution that how to manage the requirements for complex systems. Now-a-days computer systems are becoming more and more complex and projects are failing because of inadequate handling of system complexity due to lack of requirement management. They proposed an approach for requirement management. Their proposed approach is based on the requirement management tool named RequisitePro. In their papers they focused on consistency and traceability between different requirements categories and UML model also constructed. The implementation of the approach is on train system (normally a complex type of system). At the end, results have shown that if someone implements requirement management approach, the project will successful because it encompasses all types of requirement and gives a feasible solution. Eun-Ser Lee et at [4] proposed Defect opportunity tree. They made a questionnaire on requirement detection, and sent them in a company to identify the defects management opportunity tree. After the survey, they identified the Opportunity Tree items. Opportunity Tree (OT) items are (1) Requirement elicitation of risk items, (2) Requirement change, (3) Analysis requirement relationship, (4) Analysis requirement type. They give them (OT Items) rating according to defect data collection and proposed a solution which consist of (1) Requirement elicitation of risk items: maintain a checklist (2) Requirement change: Requirement Traceability (3) Analysis requirement relationship: check whether one requirement affects the other or not (4) Analysis requirement type: describe and evaluate the types of requirements.

\section{REQUIREMENT ENGINEERING}

Requirement Engineering is a process of finding the needs of stakeholders, organizing the requirements according to their priority and feasibility and documenting the requirements of the system after specification.

Software requirement engineering covers the following activities:

$\begin{array}{ll}- & \text { Feasibility studies } \\ \text { - } & \text { Requirements elicitation and analysis } \\ - & \text { Requirements validation }\end{array}$

A feasibility study checks the feasibility of the requirement of the proposed system. Whether the current system's features are possible to implement within constrains especially under specified budget, time and resource. In Requirements elicitation, when collection of requirements starts from stakeholder then find out what they really want and expect from the system. Different activities and techniques are performed during the elicitation phase like interviews, questionnaires, surveys, prototypes and brainstorming sessions etc and also it involves the end-users, different teams (Analysts, QA/QC teams, Developers),team leads, project managers, domain experts and consultants.

Requirement engineering is said to be a systematic approach. So first study the input and output of Requirement engineering process and then map with the requirement management process area of CMMI.

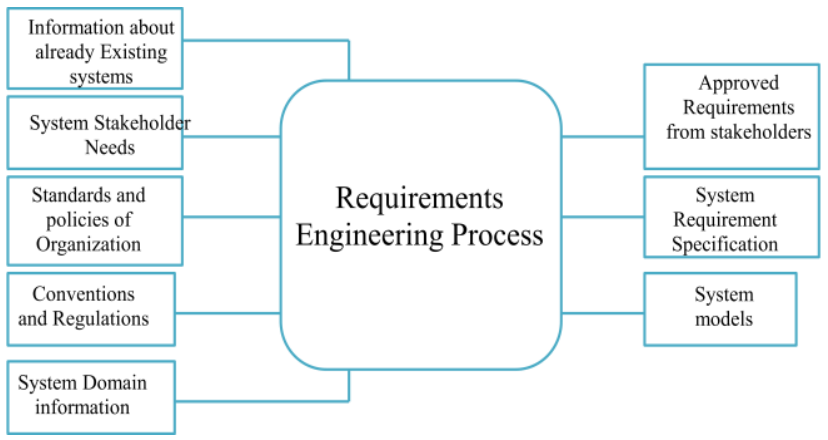

Fig1. Requirement Engineering (input/output)

In Requirement Engineering Process there are five inputs.

1. Information about already Existing System

2. System Stakeholder needs

3. Standards and policies of Organization

4. Conventions and Regulations

5. System domain information

There are three outputs of the Requirement Engineering Process.

1. Approval of requirements from stakeholders

2. System Requirement specification

3. System models

RE process inputs and outputs will map with the Requirement management Process area.

\section{REQUIREMENT MANAGEMENT CMMI PRACTICES}

CMMI (Capability Maturity Model Integration) is an approach defined by the Software Engineering Institute (SEI). It's an approach for process improvement, that helps to get better in performance of the organizations so that they can identify their strengths and weaknesses and properly avail the opportunities and can make strategies against the threats.

CMMI defined different levels by using two types of representation.

Continuous Representation: Focus on Specific Processes which are essential for fulfilling the organization's short term objectives. 
Staged Representation: organizations have to follow predefined set of process areas, managed by maturity level.

CMMI defined the 22 process area. Hierarchy of a Process area is shown in figure 2 .

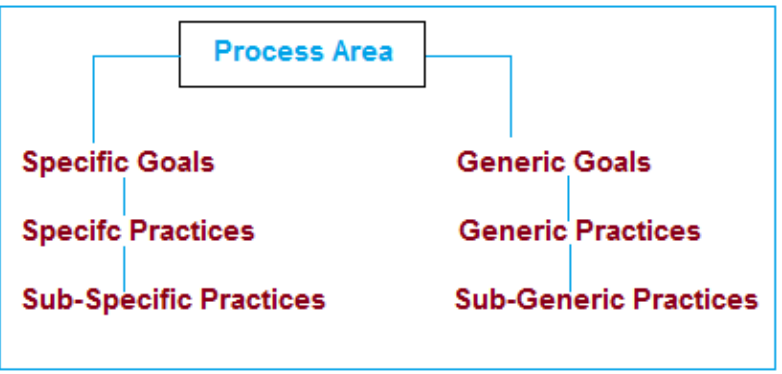

Fig 2. Process Area Structure

Requirement Management Process area is selected, which comes under the CMMI Maturity Level2. In requirement management there is a Specific Goal and five Generic Goals. Research work will map the requirement management process area with the software requirement engineering process.

\section{REQUIREMENT ENGINEERING BY USING REQUIREMENT MANAGEMENT PRACTICES}

In this section, requirement management specific goal maps with the inputs of Software requirement engineering.

Hospital management system is taken as a case study of the research.

\subsection{Information about existing system}

One should know about the existing system, also the main and sub features of the existing system. In case, a new system is required to develop, then requirement engineering process starts from inception phase.

\subsubsection{Role of Requirement Management}

Specific Practice is to acquire the understanding about the requirements of the system. Specific practice comes under the specific goal manage requirement.

\subsubsection{Application of Specific Practice}

While gathering requirements about Hospital, understand the existing process of the hospital. How they keep the record of the patients, manage their profiles, issue medicines. After understanding of the system, analyze how developer will convert the manual existing system into the user required automatic system.

\subsection{System Stakeholders Needs}

System stakeholder's needs are the main base which directs the development process. Stakeholders are those persons who have an interest in the development of the system; they may be the investor, the organizations in which the system deploys, so they can directly and indirectly interact with the system. In this activity, system requirement should reflect the stakeholder requirements.

\subsubsection{Role of Requirement Management}

Generic goal Institutionalize a Managed Process consist of generic practice named as "Identify and Involve Relevant Stakeholder". So for performing the activity of requirement engineering we should obtain the generic goal in which we identify the actual stakeholders of the system, who are the primary stakeholders, secondary stakeholders and who are the Tertiary Stakeholders (Govt. etc).Once the stakeholders are identified, now the next phase is to obtain the exact requirements from the stakeholders. Different processes will be in use to collect the requirements (needs of stakeholders). Specific Practice named as "Obtain Commitment to Requirements" will be performed, in which approval has take from the stakeholders on the requirements and agreements will be signed. If negotiation require, it will be performed.

\subsubsection{Application of Specific Practice}

Arrange meetings with the top management, Doctors and nursing staff. What they actually want, what are constrains imposed by the stakeholder like time duration and budget. How they prioritize the requirements according to their needs like patient registration module is on higher priority and issue medicine is on low priority.

\subsection{Standards and policies of an organization}

Standards and policies are used in an organization for the purpose of maintaining the consistency within an organization. Consistency means to maintain the things in stable manner. Policies are followed by the employees regarding their leaves; work etc and standard also apply on the processes which are being followed.

\subsubsection{Role of Requirement Management}

Generic practice "Establish an Organizational Policy" comes under the goal name "Institutionalize a Managed Process". Why it is need to establish the policy? Planning is required for requirement management process. Planning will only work if the some way of working will establish. Policy establishes the expectation of an organization during the requirement management process. Also it identifies that where the inconsistencies exist among different work products, or project plans, or in different departments.

\subsubsection{Application of Specific Practice}

During the development of patient registration module, different types of patients are specified, and according to their type hospital charge the fee. Hospital makes fee policy for different type of patients, old age benefits for old age patients, for poor patients there exist a policy. So understand the fee policy for the successful implementation of hospital management system and implement it according to the requirement.

\subsection{Conventions and regulations}

It includes the internal conventions and external regulations about the requirements implementations, different govt. rules 
and international level regulations should be considered while doing requirement engineering process.

\subsubsection{Role of Requirement Management}

There is no as such specific goal or generic goal specified in which the limitations on requirements according to the regulation or laws defined. But some conventions can be applied on requirements. But conventions are applied at organization level templates and predefined procedures.

\subsubsection{Application of Specific Practice}

Govt. /state regulations should be kept in mind, the system under development should not violate the laws, like issued medicines should not be expired.

\subsection{System Domain Information}

System domain information means that during requirement engineering, one should know about the system domain in which he/she will work.

\subsubsection{Role of Requirement Management}

In CMMI, system domain information comes under the "manage requirement" Specific goal and specific practices includes "Obtain an Understanding of Requirements".

Also it consist of generic practice "Identify and Involve Relevant Stakeholders" which comes under the "Institutionalize a Managed Process" generic goal.

The main input for requirement engineering is to first understand the domain of the system.

\subsubsection{Application of Specific Practice}

While working on the patient information system, so he/she should know about the working domain of Hospital, what types of patients are dealt in hospital? How their information is record? How their registrations are performing? So it is necessary to know about the Domain of the application. Entire Hospital management system comes under the Health domain, so also consider the health related issues.

\section{Future work on Requirement Management}

Future work can be done on requirement management. One can evaluate the role of requirement management in requirement engineering by specifying the internal depth details of requirement engineering. It may possible to map the different specific goals and specific practices and sub practices also generic goals, practices and sub practices with the main inputs of requirement engineering. Similarly, it can also map with the supporting inputs which provide the support to the main inputs.

\section{Conclusion}

Result of the research emphasizes on the implementation of requirement management practice during the project requirement engineering phase.

It is an efficient and effective way to adopt CMMI process area, although CMMI is a process improvement approach so when we have some support which can improve over current development process, then we should adopt that support/approach.

If requirement engineering process is not followed then it is a huge mistake which leads to inappropriate requirement engineering and at the end user needs to change the many requirements because of following causes:

- Customer not satisfied

- Budget overrun

- Time delay

- Resource unavailability

- Conflict between planned and actual requirements

Organizations should apply CMMI process improvement approach not only in the phase of requirement engineering but also in the other software development phases.

\section{References}

[1] Wang. X, Bai. Y, Cai. C and Liu. K. 2010. Research on Organizational Software Requirement Management and its Information Support. 2nd International Conference on Industrial and Information Systems Mechanics \& Electronic Engineering Faculty Shanghai Second Polytechnic University, Shanghai China.

[2] Justin J, Lin. Y and Sung. Y. 2007 . Computer Supported Cooperative Work in Design Collaborative Requirement Management System Developed for CMMI-Coherent Software Engineering. Proceedings of the 11th International Conference on Department of Industrial Engineering and Management, Chaoyang University of Technology, Taiwan.

[3] Xie. Y, Tang. T, Xu. T and Zhao. L .2010. Research on Requirement Management for Complex Systems. 2nd International Conference on Computer Engineering and Technology (ICCET), Beijing Jiaotong University(BJTU), Beijing, China.

[4] Lee. E.S and Bae. J.M. 2007. Design Opportunity Tree for Requirement Management and Software Process Improvement. International Conference on Multimedia and Ubiquitous Engineering pp395-400 . 